\title{
Synthesis and characterisation of new polymer liquid crystals for externally regulated Direct Methanol Fuel Cells.
}

\author{
A.Martinez ${ }^{1}$, P. Henderson ${ }^{2}$, E. Ballester ${ }^{1}$, C. Imrie $^{2}$ and A. Ribes ${ }^{1}$ \\ ${ }^{1}$ School of Industrial Design Engineering \\ Universidad Politecnica de Valencia \\ Camino de Vera S/N, 46022 Valencia (Spain) \\ Phone/Fax number:+0034 963879817, e-mail: aribes@ter.upv.es \\ ${ }^{2}$ Department of Chemistry, Meston Walk University of Aberdeen \\ Aberdeen AB24 3FX Aberdeen, Scotland (UK) \\ Phone/Fax number: +44 (0)1224-272000
}

\begin{abstract}
.
In this communication we present our recent work related to the design, synthesis and characterisation of new liquid crystalline materials which will be used as externally controlled electrolytes in Direct Methanol Fuel Cells (DMFC). The aim is to obtain materials with a hybrid hydrophobic / hydrophilic structure in order to minimize the "crossover" effect, which causes yield losses of up to $30 \%$ in DMFCs. By inducing liquid crystallinity to the new electrolytes, their morphology and therefore their diffusion properties against water and methanol can be externally controlled by means of electrical fields.
\end{abstract}

\section{Keywords}

Direct methanol fuel cells, crossover, bio-alcohols, liquid crystals

\section{Introduction}

Fuel cells are devices that convert chemical energy into electric power by means of electrochemical reactions [1]. The most common low temperature fuel cells use the oxidation of hydrogen in the anode to generate water and direct current:

$$
\begin{gathered}
\text { Anode: } \mathrm{H}_{2}(\mathrm{~g}) \rightarrow 2 \mathrm{H}^{+}+2 \mathrm{e}^{-} \\
\text {Cathode: } 1 / 2 \mathrm{O}_{2}+2 \mathrm{H}^{+}+2 \mathrm{e}^{-} \rightarrow \mathrm{H}_{2} \mathrm{O}
\end{gathered}
$$

The electrons are driven through an external circuit and the protons are transferred from the anode to the cathode through a polymeric electrolyte. The overall reaction leads to the formation of water, heat and electrical power:

$$
\mathrm{H}_{2}+1 / 2 \mathrm{O}_{2} \rightarrow \mathrm{H}_{2} \mathrm{O}
$$

Low temperature fuel cells are especially suitable in portable applications (vehicular, low power suppliers...).
However the use of hydrogen has some restrictions related to its obtaining, distribution and safety on board. Direct Methanol Fuel Cells (DMFCs) are a promising alternative to the use of hydrogen in fuel cells in portable applications [2]. DMFCs are supplied with an aqueous solution of methanol which is oxidised in the anode, while the oxygen from the air reacts in the cathode, according to the following reactions:

Oxidation reaction:

$$
\mathrm{CH}_{3} \mathrm{OH}+\mathrm{H}_{2} \mathrm{O} \rightarrow \mathrm{CO}_{2}+6 \mathrm{H}^{+}+6 \mathrm{e}^{-}
$$

Reduction reaction:

$$
3 / 2 \mathrm{O}_{2}+6 \mathrm{H}^{+}+6 \mathrm{e}^{-} \rightarrow 3 \mathrm{H}_{2} \mathrm{O}
$$

Overall reaction:

$$
\mathrm{CH}_{3} \mathrm{OH}+3 / 2 \mathrm{O}_{2} \rightarrow \mathrm{CO}_{2}+2 \mathrm{H}_{2} \mathrm{O}
$$

The use of liquid mixtures at the anode avoids some of the problems mentioned above related to the hydrogen fuel cells. Furthermore, methanol can be eventually obtained from renewable sources and its related technology has been successfully proved in internal engines.

The interest and investment in DMFCs have enormously increased during the last years. Their performance, however, still shows some limitations, such as some aspects related to the mass transfer at the cathode (due to the generation of $\mathrm{CO}_{2}$ ) or the yield of the oxidation reaction (poor kinetics). In particular, one of the most important problems in DMFCs is the so-called "crossover" phenomenon. Crossover occurs when the molecules of methanol flow from the anode through the electrolyte, and react with the oxygen at the cathode. This reaction generates thermal energy (heat) instead of 
electrical power, which leads to a decrease in the efficiency of the cell (crossover can cause a drop of up to the $30 \%$ of the cell yield) [3]

In order to minimize the crossover it is paramount to design electrolytes with very specific properties. The polymeric membranes used in DMFC as electrolytes must posses high proton conductivities and relatively high water contents, but also low methanol permeabilities. The commercial membranes used in $\mathrm{H}_{2}$ fuel cells are not suitable to DMFC because of their high methanol solubility (and crossover). As a consequence, different materials have been designed during the last years for their specific use in DMFC. However, attempts to decrease the methanol permeation usually lead to a decrease in the water contents in the membrane, and a proton conductivity drop, since the transport mechanisms of all of them are strongly related.

The research in the field of new materials in DMFC has been recently focused on the design of materials with a hydrophobic backbone and hydrophilic groups with high proton affinities. The aim is to discriminate the diffusion mechanisms of water, methanol and the proton conductivity.

In this work we present the design, synthesis and characterization of new hydrophobic / hydrophilic membranes based in liquid crystal polymers for their use as electrolytes in DMFCs. With the presence of liquid crystalline groups, the resulting membranes can be externally controlled in the mesomorphic range. This provides a mechanism to control the resulting morphology of the polymer, which plays a determinant role in the diffusion of methanol and water and also in the proton conductivity. By varying the phase separation rate between hydrophilic and hydrophobic domains a more accurate control of the crossover phenomenon is expected.

\section{Materials}

Different materials have been synthesized and characterised prior to their use as electrolytes in DMFCs. In particular, two series of liquid crystal polymers (LCPs) have been studied.

The first set of materials was based on a poly(vinyl alcohol) (PVA) backbone containing liquid crystal moieties. PVA has been extensively used during the last years as pervaporation membranes in applications related to the separation of water and alcohol mixtures [4]. Several crosslinked PVA membranes were prepared by using sulfosuccinid acid (SSA) as crosslinking agent (figure 1). The sulfonic group in SSA provides high protonic conductivities to the membranes:

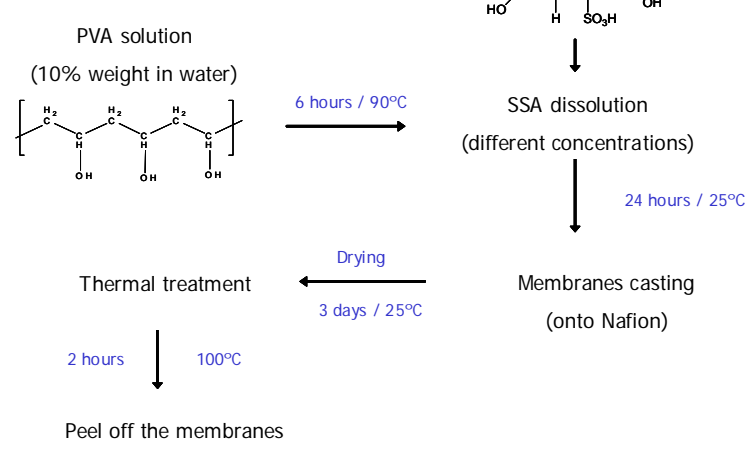

Figure 1. Preparation of the PVA - SSA membranes

In order to promote the morphology control in these membranes, different liquid crystal molecules were attached by esterification.

The liquid crystals used in this kind of polymers were 4pentoxybenzoic acid and 6- [4-(4methoxyphenylazo)phenoxy]hexanoic acid. Commercial 99\% hydrolysed PVA was used. The esterification proceeded in pyridine and homogeneous phase (Einhorn esterification) [5] as shown in figure 3:
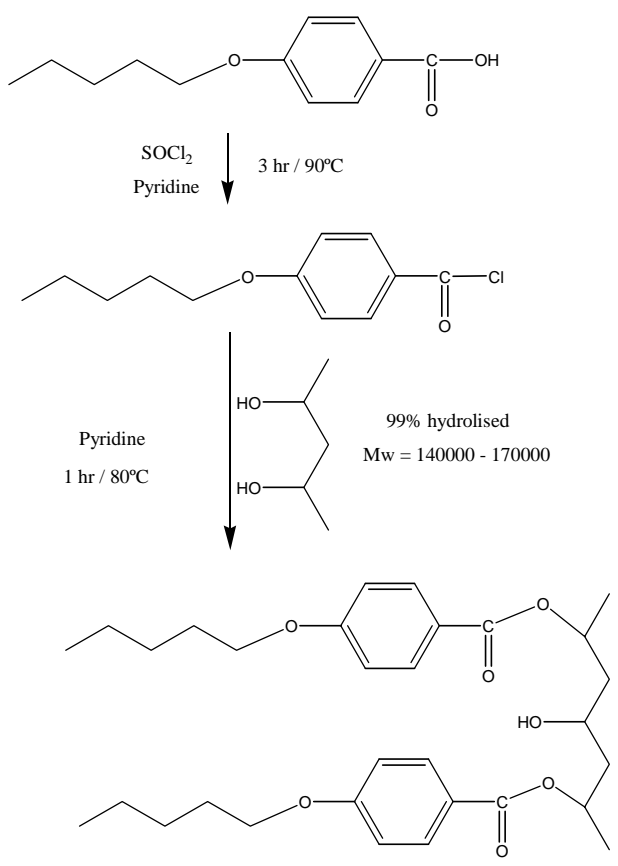

Figure 3. PVA - liquid crystals synthetic route

An alternative series of materials is based on methacrylates liquid crystalline polymers (LCP). Copolymers of 10-(4-methoxy-4'-oxy-azobenzene) decyl methacrylate and 2-acrylamido-2-methyl-1-propane sulfonic acid (AMPS) were synthesised by means of free radical polymerisation [6]. The structure of the polymers is shown in figure 4. 


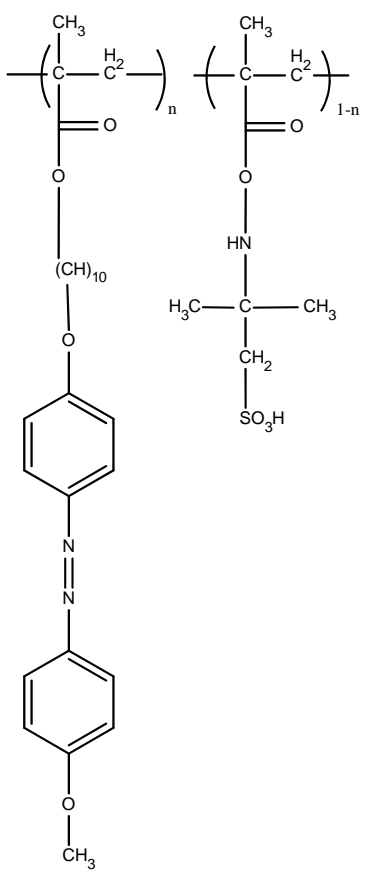

Figure 4. Structure of the methacrylates-based liquid crystal polymers.

Different co-polymers were prepared by varying the composition of the starting monomers. DMF was used as the medium of the reaction, which was performed in nitrogen atmosphere during 24 hours. The composition ranged from 0 to $100 \%$ in molar percentage of AMPS, although the production was focused in the polymers with high concentrations of the liquid crystal monomer (lower concentrations inhibit the mesomorphic behaviour)

In order to enhance the mechanical properties of the membranes, the resulting polymers were spread between two films of non-woven polypropilene (PP). The copolymers and the PP films were then melted at high pressure and then cooled down to obtain self standing films.

\section{Morphological characterisation}

The different materials and membranes were characterised by means of Fourier Transform Infrared Spectroscopy (FTIR), Differential Scanning Calorimetry (DSC) and Thermogravimetric Analysis (TGA) in order to study its physical properties.

FTIR gives valuable information about the functional groups present in the polymer. This includes studies about the water contents, the interactions between the hydrophobic and hydrophilic segments, etc. The presence of proton conductive groups can also be detected by the use of FTIR. The study of the IR spectra of the polymers was focused in particular bands of absorption with high interest. This is the case of the sulfonic stretching vibrations (at $1057 \mathrm{~cm}^{-1}$ and $983 \mathrm{~cm}^{-1}$ ) or the $\mathrm{C}=\mathrm{O}$ vibration region $\left(1720 \mathrm{~cm}^{-1}\right)$. The study of the absorption values, possible shifts of the bands and area gave valuable information about the synthesis process and also the chemical environment of the functional groups. FTIR was also used to evaluate the effect of some of the diffusion tests in the components of the polymer.

DSC is used to study the different thermal transitions of the materials. It is commonly used to characterise the liquid crystals mesophases through the calculation of the temperatures and enthalpies of the transitions. DSC can be very useful to study the water and methanol contents of the materials as well as the chemical nature of the solvents (free water, bound water...) Another important aim of the DSC tests is to study the variations of the polymeric properties in the co-polymers (glass transitions, crystallinity, etc) since they can seriously affect the water and methanol transport. In particular, the formation of clusters in the ionic (hydrophilic) domains can be displayed if the thermal transitions are properly reported.

Finally, TGA is used to characterise the thermal stability of the materials. Even though DMFCs usually operate at low temperatures (below $100^{\circ} \mathrm{C}$ ) it is important to assure that the thermal properties of the polymers have not been affected during the synthesis and preparation of the membranes. Dynamic and isothermal tests are carried out to the materials in order to simulate their use in DMFC.

\section{Diffusion characterisation}

As it was mentioned above, in order to reduce "crossover" in DMFC electrolytes it is important to discriminate the diffusion of methanol, water and the proton conductivity. Therefore, it is paramount to analyse the diffusivity properties of the different LCPs and to compare them with the commercial materials in different conditions. This is carried out in our lab by means of swelling, pervaporation and externally controlled tests.

The first stage to study the permeability of the membranes respect to water and methanol are the socalled swelling tests. In swelling tests small samples are submerged in solutions of methanol and water with different concentrations during long immersion times at a controlled temperature. The solvent absorption is then measured gravimetrically as a function of the time (see figure 5).

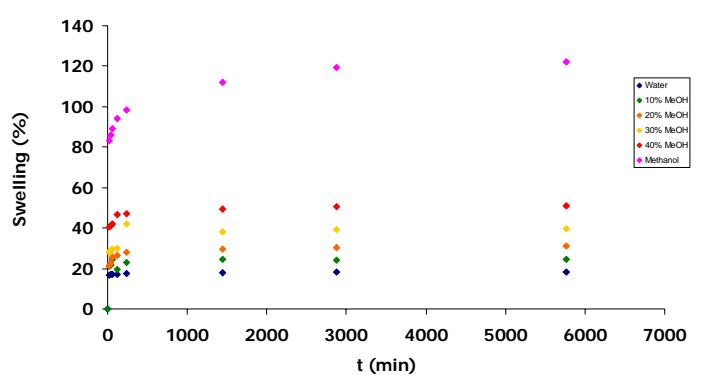

Figure 5. Example of swelling curves at different methanol concentration 
The results obtained from the swelling tests give valuable information about the solubility of the solvents in the membranes, the kinetics of diffusion and even about the preferential absorption of the components in the mixtures.

After the equilibrium is reached, the physicochemical properties of the samples are analysed by means of the techniques explained in section 3. The results are then compared with those corresponding to the samples before the swelling experiments. The study can provide information about the interactions between the polymeric chain and the solvents, can also complete the results related to preferential absorption and analyse the effect of the temperature in the diffusivity. As an example, FTIR can be used to determine the chemical environment of the solvents in the steady state by studying the $3000 \mathrm{~cm}^{-1}$ region (OH vibration), but also to search for possible effects in the chemical environment of the polymer chains (see figure 6)

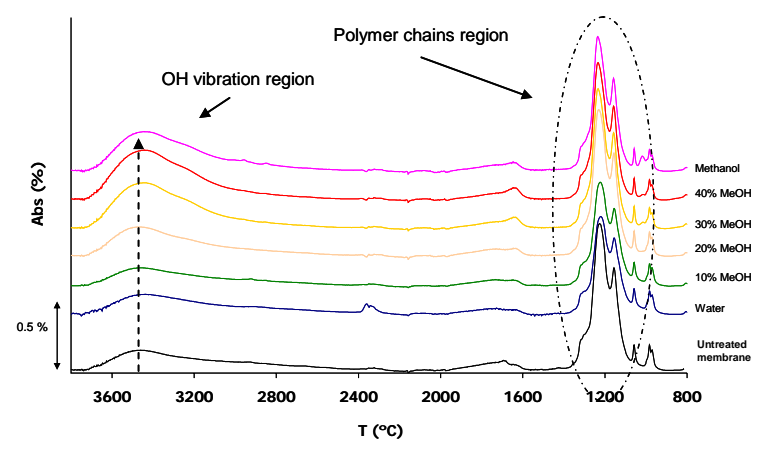

Figure 6. Scheme of the modulus to test externally controlled materials

A more accurate approach to the role of the membranes in DMFC is carried out in the pervaporation tests. In such tests the diffusion properties of the membranes against water and alcohol are studied in the vapour phase. The compounds are forced to vaporise by applying a very low pressure on the permeate side of the membrane. The driving force for the separation is then the difference in the partial pressures $\left(\Delta \mathrm{P}_{\mathrm{i}}\right)$ of the components on the two sides of the membrane. Therefore, the separation of the components is based on a difference in the transport rate of individual components through the membrane.

A self-designed pervaporation plant was used to perform the tests. A picture of the plant can be seen in the following figure 7:

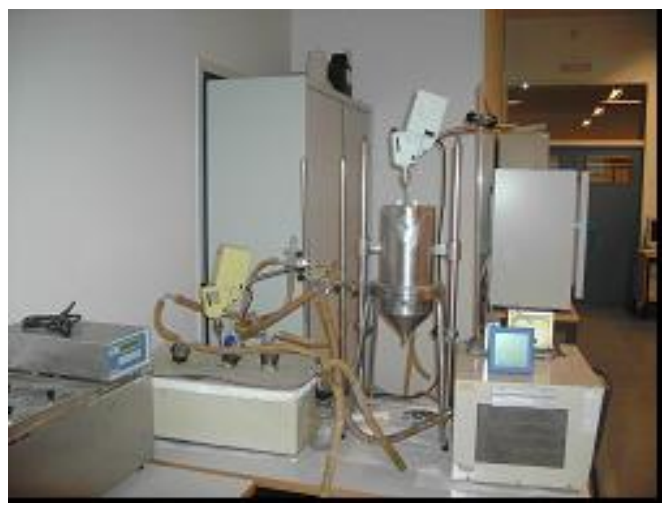

Figure 7. Picture of the pervaporation plant.

The alcohol solution is contained in the upper part of the plant, in contact with one of the sides of the membrane, and is controlled by using a recirculation bath. The pervaporation tests were carried out at different temperatures. A vacuum pump is connected to the permeate side of the plant, and the vaporisation occurs depending on the vapour pressure of the components. After transferring through the membrane, the permeate (in the vapour phase) is forced to pass through three compartments which are kept at very low temperature (around $-90^{\circ} \mathrm{C}$ ) with the help of an intracooler. The permeate mixture condenses in the balloons and is collected as a function of time.

The diffusivity of liquid mixtures of water and methanol is measured at atmospheric pressure by using a self designed separation plant. A picture of the central part of the modulus can be seen in figure 8:

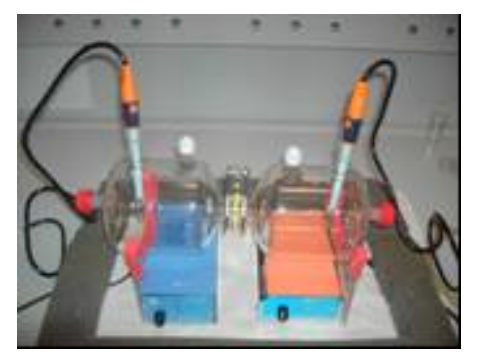

Figure 8. Picture of the two tanks used in the diffusion plant

The central part of the plant consists of two rounded glassy tanks of 1 litter capacity each between which the membrane is placed. One of the tanks contains the water/alcohol solution (simulating the anode fuel stream) while the other contains only pure water (simulating the cathode conditions). The temperature is controlled by two cylindrical resistors and the concentration in the tanks is monitored by conductivity.

Two iron mesh electrodes are located on both sides of the membrane to apply different electrical fields. The application of the electrical field promotes the polarisation of the liquid crystal moieties and a change in the morphology of the polymer, but also simulates the electrical conditions which cause the electroosmotic drag 
of methanol and water from the anode to the cathode in DMFC.

A scheme of the plant is shown in figure 9:

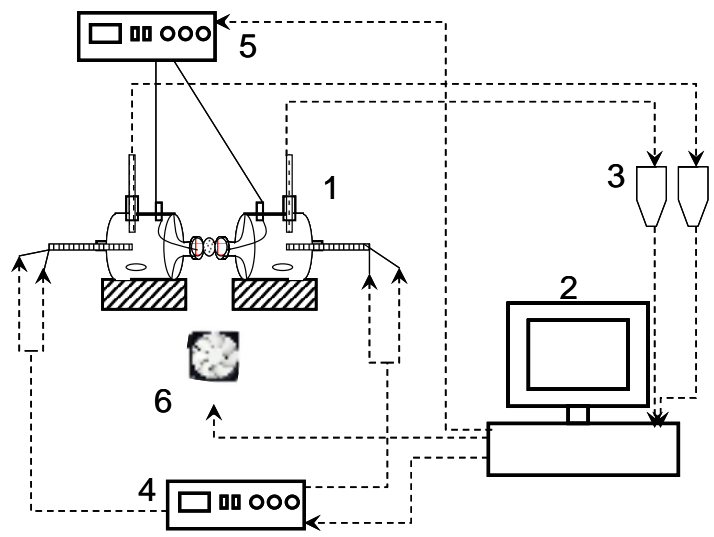

Figure 9. Scheme of the modulus to test externally controlled materials (legends in text).

An Acer personal computer (PC) with an acquisition card (PCI 9112) is used to collect the data and control the plant (2). Two conductimeters CON110 provided by Eutech Instruments are used to measure conductivity and temperature (3). The cell constant for the experiments is $\mathrm{K}=1$, and the temperature accuracy $\pm 0.1^{\circ} \mathrm{C}$. The resistors and the electrodes were powered by two Grelco 6VD305SF power supplies $(4,5)$ in a voltage range of 0 $-30 \mathrm{~V}$ and an intensity range of $0-5 \mathrm{~A}$. The system is controlled by a LabView program.

The diffusivity of methanol is measured at different temperatures. The methanol diffuses from one side to the other of the membrane and the conductivity is measured as a function of time. The diffusivity of the methanol can be calculated from the slopes of the curves:

$$
C_{B}(t)=\frac{A}{V_{B}} \frac{D K}{L} C_{A}\left(t-t_{0}\right)
$$

where $C_{B}$ and $C_{A}$ are the methanol concentrations in the tanks and $A$ and $L$ are the membrane area and thickness, respectively. $D$ is the methanol diffusivity of the membrane, and $K$ the partition coefficient between the membrane and the adjacent solution. The permeability $P$ can be calculated as $P=D K$ if $D$ is considered homogeneous inside the membrane and $K$ independent of the concentration of methanol.

\section{Conclusions}

Several materials which are potential new electrolytes for Direct Methanol Fuel Cells (DMFC) are being synthesized and investigated. The new materials contain liquid crystal polymers in their structure. The aim is to control the diffusion of methanol, water and the proton conductivity by changing the morphology of the mesophases. The transport properties can be altered by varying the hydrophobic / hydrophilic phase separation caused by the presence of a scarcely polar backbone and proton conducting groups (usually ionic). A methodology for characterising the physicochemical properties of the materials is also presented. Several techniques of thermal analysis and spectroscopy are used in conjunction with diffusion tests. The transport properties of the membranes against water and methanol mixtures are determined by swelling and pervaporation tests and also using a selfmade plant to test externally controlled materials, simulating the conditions of a real DMFC.

\section{Acknowledgement}

The authors would like to acknowledge the Spanish Ministry of Science and Innovation for its support through the Research Project ENE2007-67584-C03-02 and one FPU predoctoral grants.

\section{References}

[1] L. Blomen, Fuel Cell Systems, Ed. Plenum Press, New York, (1993)

[2] P.J. Collings and M. Hird, Introduction to Liquid Crystals: Chemistry and Physics, Ed. Taylor \& Francis, New York (1994)

[3] Woong J.C., Venkataramani S., Kim S.C., "Modification of Nafion membrane using poly(4-vinyl pyridine) for direct methanol fuel cell” Polym. Internat. Vol 55 (2006), pp 491 - 499

[4] J.W. Rhim, H. B. Park, C.S. Lee, J.H. Jun, D.S. Kim, Y.M. Lee, Journal of Membrane Science, "Crosslinked poly(vinyl alcohol) membranes containing sulfonic acid group: proton and methanol transport through membranes” 238 (2004) pp 143 - 151.

[5] Janietz, D; Bauer, M, "Chromophoric poly(vinyl alcohol derivative)s .1. Synthesis and spectroscopical characterization of some poly(vinyl alcohol)s with alkoxyazobenzenecarbonyl substituents" Makromolecular Chemie., 192 (1991) pp 2635 - 2640

[6] A. Cook, C. Imrie, "Side-Group Liquid Crystal copolymers containing nonmesogenic units: dependence of the thermal behaviour upon concentration” Molecular Crystals Liquid Crystals, Vol 332 (1999) pp 189 - 198 